\title{
INSIGHTS ON THE STELLAR MASS-METALLICITY RELATION FROM THE CALIFA SURVEY
}

\author{
R. M. González Delgado ${ }^{1}$, R. Cid Fernandes ${ }^{2}$, R. García-Benito ${ }^{1}$, E. Pérez ${ }^{1}$, A. L. de Amorim ${ }^{2}$, C. Cortijo-Ferrero ${ }^{1}$, \\ E. A. D. Lacerda ${ }^{2}$, R. López Fernández ${ }^{1}$, S. F. SÁnchez ${ }^{1,3,4}$, N. Vale Asari ${ }^{2}$, J. Alves ${ }^{5}$, J. Bland-Hawthorn ${ }^{6}$, \\ L. Galbany ${ }^{7}$, A. Gallazzi ${ }^{8,9}$, B. Husemann ${ }^{10,11}$, S. Bekeraite ${ }^{11}$, B. Jungwiert ${ }^{12}$, A. R. López-Sánchez ${ }^{13}$, \\ A. De Lorenzo-Cáceres ${ }^{14}$, R. A. Marino ${ }^{15}$, D. Mast ${ }^{16}$, M. Mollá $^{17}$, A. Del Olmo ${ }^{1}$, P. SÁnchez-Blázquez ${ }^{18}$, \\ G. VAN DE VEN ${ }^{19}$, J. M. VílCheZ ${ }^{1}$, C. J. WALCher ${ }^{11}$, L. Wisotzki ${ }^{11}$, B. Ziegler ${ }^{5}$, AND CALIFA Collaboration ${ }^{9,20}$ \\ ${ }^{1}$ Instituto de Astrofísica de Andalucía (CSIC), Glorieta de la Astronomía s/n, E-18008 Granada, Spain \\ ${ }^{2}$ Departamento de Física, Universidade Federal de Santa Catarina, P.O. Box 476, 88040-900 Florianópolis, SC, Brazil \\ ${ }^{3}$ Centro Astronómico Hispano Alemán, Calar Alto (CSIC-MPG), Jesús Durbán Remón 2-2, E-04004 Almería, Spain \\ ${ }^{4}$ Instituto de Astronomía,Universidad Nacional Autonóma de Mexico, A.P. 70-264, 04510 Distrito Federal, Mexico \\ ${ }^{5}$ University of Vienna, Türkenschanzstrasse 17, A-1180 Vienna, Austria \\ ${ }^{6}$ Sydney Institute for Astronomy, The University of Sydney, NSW 2006, Australia \\ ${ }^{7}$ Millennium Institute of Astrophysics and Departamento de Astronomía, Universidad de Chile, Casilla 36-D, Santiago, Chile \\ ${ }^{8}$ INAF-Osservatorio Astrofisico di Arcetri, Largo Enrico Fermi 5, I-50125 Firenze, Italy \\ ${ }^{9}$ Dark Cosmology Center, University of Copenhagen, Niels Bohr Institute, Juliane Maries Vej 30, DK-2100 Copenhagen, Denmark \\ ${ }^{10}$ European Southern Observatory, Karl-Schwarzschild-Strasse 2, D-85748 Garching b. München, Germany \\ ${ }^{11}$ Leibniz-Institut für Astrophysik Potsdam, An der Sternwarte 16, D-14482 Potsdam, Germany \\ 12 Astronomical Institute of the Academy of Sciences of the Czech Republic, v.v.i., Bocni II 1401, 14131 Prague, Czech Republic \\ ${ }^{13}$ Australian Astronomical Observatory, P.O. Box 915, North Ryde, NSW 1670, Australia \\ ${ }^{14}$ School of Physics and Astronomy, University of St. Andrews, North Haugh, St. Andrews, KY16 9SS, UK \\ ${ }^{15}$ CEI Campus Moncloa, UCM-UPM, Departamento de Astrofísica y CC. de la Atmósfera, Facultad de CC. Físicas, \\ Universidad Complutense de Madrid, Avda. Complutense s/n, E-28040 Madrid, Spain \\ ${ }^{16}$ Instituto de Cosmologia, Relatividade e Astrofísica ICRA, Centro Brasileiro de Pesquisas Físicas, Rua Dr. Xavier Sigaud 150, \\ CEP 22290-180, Rio de Janeiro, RJ, Brazil \\ ${ }^{17}$ Departamento de Investigación Básica, CIEMAT, Avda. Complutense 40, E-28040 Madrid, Spain \\ ${ }_{18}^{18}$ Depto. de Física Teórica, Universidad Autónoma de Madrid, E-28049 Madrid, Spain \\ ${ }^{19}$ Max-Planck-Institut für Astronomie, Königstuhl 17, D-69117 Heidelberg, Germany \\ ${ }^{20}$ CALIFA International Collaboration http://califa.caha.es \\ Received 2014 June 18; accepted 2014 July 3; published 2014 July 28
}

\begin{abstract}
We use spatially and temporally resolved maps of stellar population properties of 300 galaxies from the CALIFA integral field survey to investigate how the stellar metallicity $\left(Z_{\star}\right)$ relates to the total stellar mass $\left(M_{\star}\right)$ and the local mass surface density $\left(\mu_{\star}\right)$ in both spheroidal- and disk-dominated galaxies. The galaxies are shown to follow a clear stellar mass-metallicity relation (MZR) over the whole $10^{9}-10^{12} M_{\odot}$ range. This relation is steeper than the one derived from nebular abundances, which is similar to the flatter stellar MZR derived when we consider only young stars. We also find a strong relation between the local values of $\mu_{\star}$ and $Z_{\star}$ (the $\mu \mathrm{ZR}$ ), betraying the influence of local factors in determining $Z_{\star}$. This shows that both local $\left(\mu_{\star}\right.$-driven) and global $\left(M_{\star}\right.$-driven) processes are important in determining metallicity in galaxies. We find that the overall balance between local and global effects varies with the location within a galaxy. In disks, $\mu_{\star}$ regulates $Z_{\star}$, producing a strong $\mu$ ZR whose amplitude is modulated by $M_{\star}$. In spheroids it is $M_{\star}$ that dominates the physics of star formation and chemical enrichment, with $\mu_{\star}$ playing a minor, secondary role. These findings agree with our previous analysis of the star formation histories of CALIFA galaxies, which showed that mean stellar ages are mainly governed by surface density in galaxy disks and by total mass in spheroids.
\end{abstract}

Key words: galaxies: evolution - galaxies: fundamental parameters - galaxies: stellar content - galaxies: structure

Online-only material: color figures

\section{INTRODUCTION}

The quest for understanding the physical association between mass and metallicity in galaxies has a long history in astrophysics. In its currently most common form, mass is represented by the stellar mass $\left(M_{\star}\right)$, while the nebular oxygen abundance is taken as the metallicity tracer. Perhaps the best known contemporary example of this mass-metallicity relation (MZR) is that derived for star-forming galaxies in the Sloan Digital Sky Survey (SDSS) by Tremonti et al. (2004).

An independent way to explore the metal content of galaxies is through their stars, whose combined spectra encode a fossil record of galaxy evolution. This approach has been widely explored in relation to elliptical galaxies, which also follow a
MZR (the Mg- $\sigma_{\star}$ relation; Faber \& Jackson 1976). The historical limitation to early-type systems comes about because of their predominantly old and relatively simple stellar populations (SSPs, often approximated as a single burst), in contrast with the more complex star formation histories (SFHs) of spirals, which complicates the translation of their spectroscopic features into tracers of the characteristic stellar metallicity $\left(Z_{\star}\right)$. Recent work has started to lift this restriction. In particular, spectral synthesis techniques have shown that useful estimates of $Z_{\star}$ can be derived for galaxies of all types (Cid Fernandes et al. 2005, 2007; Gallazzi et al. 2005; Panter et al. 2007), especially when used comparatively and for large samples. These studies obtain a purely stellar version of the MZR, as well as a broad correlation between $\mathrm{O} / \mathrm{H}$ and $Z_{\star}$, and a $Z_{\star}$ evolutionary pattern 
characteristic of chemical enrichment, all derived on the basis of an archeological spectral analysis of local SDSS galaxies.

A common problem in both approaches is that they are most often based on integrated data, where one gathers all or part of the light of a galaxy in a single spectrum. The exact meaning of the global $\mathrm{O} / \mathrm{H}, Z_{\star}$, and other properties derived from such data is rendered somewhat ambiguous (and aperturedependent; e.g., Iglesias-Páramo et al. 2013; Mast et al. 2014) by the existence of spatial gradients in both nebular and stellar population properties within galaxies. More critically, the lack of spatial resolution limits our ability to map the influence of local factors on the local metallicity which, if present, propagate to the global MZR.

Integral field spectroscopy surveys like CALIFA (Sánchez et al. 2012) best tackle these issues. Rosales-Ortega et al. (2012) and Sánchez et al. (2013) applied nebular diagnostics to thousands of $\mathrm{H}$ II regions in these data cubes, finding that $\mathrm{O} / \mathrm{H}$ is strongly correlated with the local stellar mass surface density $\left(\mu_{\star}\right)$, and that this relation shapes the global MZR. The notion that $M_{\star}$ plays a less fundamental role than $\mu_{\star}$ in the evolution of galactic disks (homes of the $\mathrm{H}$ II regions whose $\mathrm{O} / \mathrm{H}$ represent the metallicity in the nebular MZR) has been raised previously. Bell \& de Jong (2000), for instance, analyzed optical and nearIR imaging of spirals, finding that the surface density of a galaxy drives its $\mathrm{SFH}$, and that $M_{\star}$ is a less important parameter. In the context of integral field studies, González Delgado et al. (2014, hereafter GD14) carried out a spectral synthesis analysis of 107 CALIFA galaxies, also finding that mean stellar ages correlate more strongly with $\mu_{\star}$ in galaxy disks. Conversely, they find that in spheroids (elliptical galaxies and bulges), it is $M_{\star}$ that controls the $\mathrm{SFH}$.

Here we explore global and local estimates of the $Z_{\star}$ obtained from the spatially resolved spectral synthesis of CALIFA galaxies. The focus on stars complements the work based on H II regions, bringing in new perspectives. First, stellar metallicities reflect the whole history of a galaxy, while $\mathrm{O} / \mathrm{H}$ measures a present-day snapshot of its evolution. Second, our analysis ignores $\mathrm{H}$ II regions, and so can be applied to disks and spheroids, increasing statistics and broadening the scope of the study.

\section{DATA AND STELLAR POPULATION ANALYSIS}

Our sample comprises 300 galaxies ranging from ellipticals to late-type spirals, representative of the full CALIFA mother sample (Walcher et al. 2014). Technical details are described in Sánchez et al. (2012), Husemann et al. (2013), and the forthcoming Data Release 2 paper by R. García-Benito et al. (in preparation).

Our method to extract stellar population properties from data cubes has been explained in Pérez et al. (2013) and Cid Fernandes et al. (2013, 2014). In short, we use STARLIGHT (Cid Fernandes et al. 2005) to fit each spectrum as a combination of SSPs spanning different ages and metallicities. The SSP spectra used in this work consist of Granada (González Delgado et al. 2005) and MILES (Vazdekis et al. 2010) models, similar to those used in Cid Fernandes et al. (2013) but extended to cover the full metallicity range of the MILES models. The 235 elements base covers ages from 0.001 to $14 \mathrm{Gyr}$, and $\log Z_{\star} / Z_{\odot}$ from -2.3 to +0.33 . A Salpeter initial mass function is adopted.

This whole analysis transforms the data cubes to twodimensional maps of a series of stellar population properties. The main ones for the purposes of this Letter are the stellar mass surface density, and the mass-weighted mean stellar metallicity, defined as

$$
\left\langle\log Z_{\star}\right\rangle_{M, x y}=\frac{\sum_{t Z} M_{\star, t Z, x y} \times \log Z}{\sum_{t Z} M_{\star, t Z, x y}},
$$

where $M_{\star, t Z, x y}$ denotes the mass in stars of age $t$ and metallicity $Z$ in spaxel $x y$.

\section{RESULTS}

\subsection{The Global Mass-Metallicity Relation}

The MZR is a relation between global galaxy properties, which requires us to compress our $x y$ maps to single numbers. The total stellar mass is obtained from $M_{\star}=\sum_{x y} M_{\star, x y}$, i.e., adding up the masses derived for individual spaxels (thus accounting for spatial variations in the mass-to-light ratio and extinction). For the metallicity, we use a galaxy-wide averaging scheme, such that

$$
\left\langle\log Z_{\star}\right\rangle_{M}^{\text {galaxy }}=\frac{\sum_{x y} M_{\star, x y}\left\langle\log Z_{\star}\right\rangle_{M, x y}}{\sum_{x y} M_{\star, x y}},
$$

as done for light and mass-weighted mean stellar ages by GD14, who showed that these averages match very well the value obtained from the synthesis of the integrated spectrum, as well as that at radial distances of $R=1$ half light radius (HLR).

Our mass versus stellar metallicity relation for CALIFA galaxies is shown in Figure 1(a). A clear correlation is seen, with metallicities rising by roughly an order of magnitude for $M_{\star}$ between $\sim 10^{9}$ and $10^{12} M_{\odot}$. The thick black curve, obtained by smoothing the mass-binned relation, offers as better visualization of the general trend. In order to get a sense of the SFHs in this plane, we color-code the points by the value of the galaxy-wide mean stellar age (see Equation (1) of GD14). One sees that galaxies become progressively older as one moves up the MZR.

Figure 1 also compares our stellar MZR with those obtained for SDSS galaxies by Gallazzi et al. (2005; brown line) and Panter et al. (2008; magenta), both shifted by +0.25 dex in mass to match our initial mass function. Given the significant differences in data, samples, and methodology, one should not take this comparison too literally. The overall agreement, however, is remarkable.

\subsection{Stellar Versus Nebular MZRs and Chemical Evolution}

The flattening of our MZR at $\sim 10^{11} M_{\odot}$ is reminiscent of the behavior observed in the nebular MZR (e.g., Tremonti et al. 2004). A quantitative comparison of nebular and stellar metallicities is not warranted given the huge differences in the underlying physics, not to mention inherent uncertainties affecting both estimates. A qualitative comparison, however, is instructive. Figure 1(b) overplots the Sánchez et al. (2013; cyan line) relation between $M_{\star}$ and $\mathrm{O} / \mathrm{H}$ obtained from $\mathrm{H}$ II regions in CALIFA data cubes. The two (black and cyan lines) occupy the same zone at high $M_{\star}$, but diverge at low $M_{\star}$, with the nebular MZR being visibly flatter than the stellar one.

Besides the caveats noted above, this comparison is skewed by the fact that $\mathrm{O} / \mathrm{H}$ portrays the current state of the warm gas, while $Z_{\star}$ reflects the whole history of a galaxy. To mitigate this mismatch in timescales, we compute $Z_{\star}$ considering only stars younger than 2 Gyr, and derive the corresponding galaxy-wide average. The resulting MZR is shown as blue stars in Figure 1(b), with a thick blue line representing its smoothed version. 

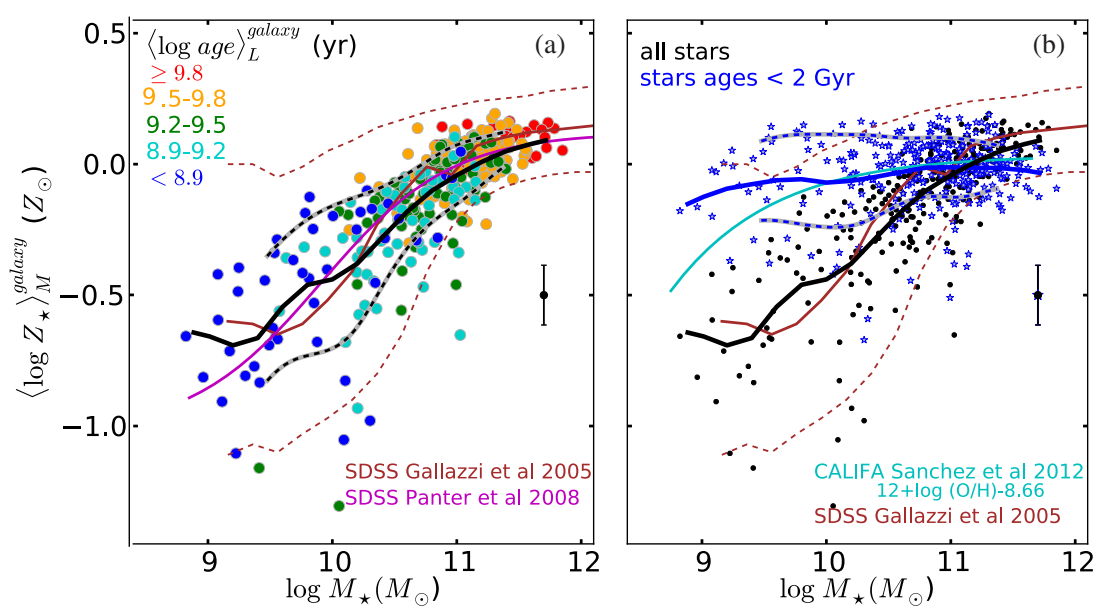

Figure 1. (a) Global stellar MZR for 300 CALIFA galaxies is shown as circles, color-coded by the mean stellar age. A mass-binned smooth mean relation is shown as a black solid line (the dashed black indicates the 16th and 84th percentiles, and the error bar represents the typical dispersion). The MZRs obtained for SDSS galaxies by Gallazzi et al. (2005) and Panter et al. (2008) are plotted as brown and magenta lines, respectively, with a dashed brown line indicating the 16th and 84th percentiles of Gallazzi et al. (b) Comparison of our global stellar MZR (black circles and line) with the one obtained considering only stars younger than 2 Gyr in the computation of the stellar metallicity (blue stars and line); blue dashed lines indicate the 16th and 84th percentiles; error bar represents the typical dispersion. The CALIFA-based nebular MZR (Sánchez et al. 2013) is shown as a cyan line.

(A color version of this figure is available in the online journal.)
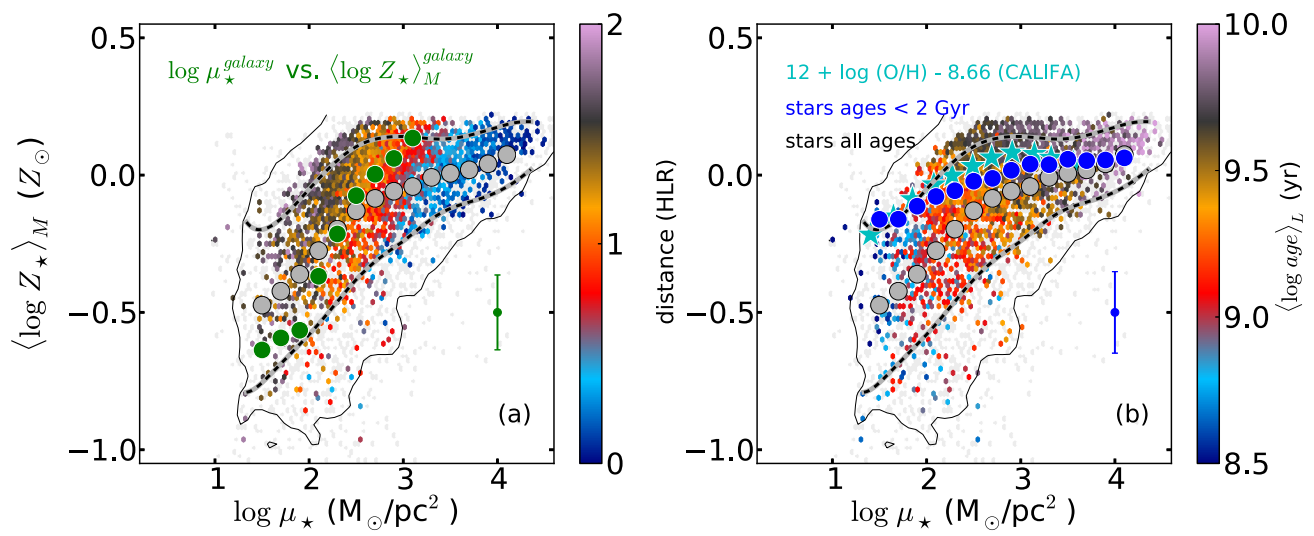

Figure 2. Local stellar metallicity vs. the local stellar mass surface density. Small dots show values for 6000 radial bins of 300 galaxies. The solid line traces the contour of point number density larger than 1 per $0.075 \times 0.035$ dex box. (a) Gray circles track the $\mu_{\star}$-binned average $Z_{\star}\left(\mu_{\star}\right)$ relation (black dashed lines indicate the 16 th and 84th percentiles). Green circles trace the $\left(\mu_{\star}\right.$-binned) global relation, obtained from the $\left\langle\log Z_{\star}\right\rangle_{M}^{\text {galaxy }}$ and $\mu_{\star}^{\text {galaxy }}$ galaxy-wide averages; the error bar represents the typical dispersion in this relation. The individual points are color-coded by the distance from the center (in HLR). (b) Dots as in panel (a), but colored to display the (luminosity-weighted) mean stellar age. Large blue circles show the $\mu_{\star}$-binned global relation obtained considering only stars younger than $2 \mathrm{Gyr}$ in the computation of $Z_{\star}$ (error bar represents the typical dispersion). Cyan stars show the CALIFA-based nebular $\mu$ ZR of Sánchez et al. $(2013)$, assuming $12+\log (\mathrm{O} / \mathrm{H}) \odot=8.66$.

(A color version of this figure is available in the online journal.)

The plot reveals a clear signature of chemical evolution, with young stars being more enriched than older stars. The MZR for young stars is flatter than the global one, in line with the nebular relation.

The overall picture portrayed by Figure 1 is the same as that drawn by Cid Fernandes et al. (2007) in their analysis of SDSS galaxies: most of the chemical evolution of massive galaxies has occurred many gigayears ago, while low- $M_{\star}$ systems grow their metallicities over a longer cosmic time span. This "chemical downsizing" is, of course, not coincidental, since star formation and chemical evolution work in tandem.

\subsection{The Local and Global $\mu_{\star}$ Versus $Z_{\star}$ Relations}

Our data are ideally suited to investigate the relative roles of global and local properties in controlling the metallicity. To explore the information encoded in our spatially resolved maps of stellar population properties, we first compress them to one-dimensional radial profiles computed as explained in GD14. Besides allowing for clearer visualization of the results, this compression (1) reduces statistical uncertainties (see Cid Fernandes et al. 2013), and (2) balances the role of each galaxy in our statistics, which would otherwise be biased toward galaxies filling up more spaxels in the integral field unit.

Radial profiles of $\mu_{\star}$ and $\left\langle\log Z_{\star}\right\rangle_{M}$ were computed in elliptical annuli in steps of $0.1 \mathrm{HLR}$. The profiles typically cover out to $R=2$ HLR reliably, so we restrict our analysis to this limit.

Figure 2(a) shows the resulting $\mu_{\star}-Z_{\star}$ relation ( $\mu \mathrm{ZR}$ ). Note that this plot is ultimately a collection of 300 metallicity profiles, where the radial coordinate is replaced by $\mu_{\star}(R)$, such that $R$ increases to the left. The $\sim 6000$ dots ( 20 per galaxy) are colorcoded according to their distance from the nucleus, in units of the corresponding HLR. Points in the upper right correspond to the inner regions of massive, early-type spheroids, while in the bottom left the outer zones of late-type spirals dominate. 

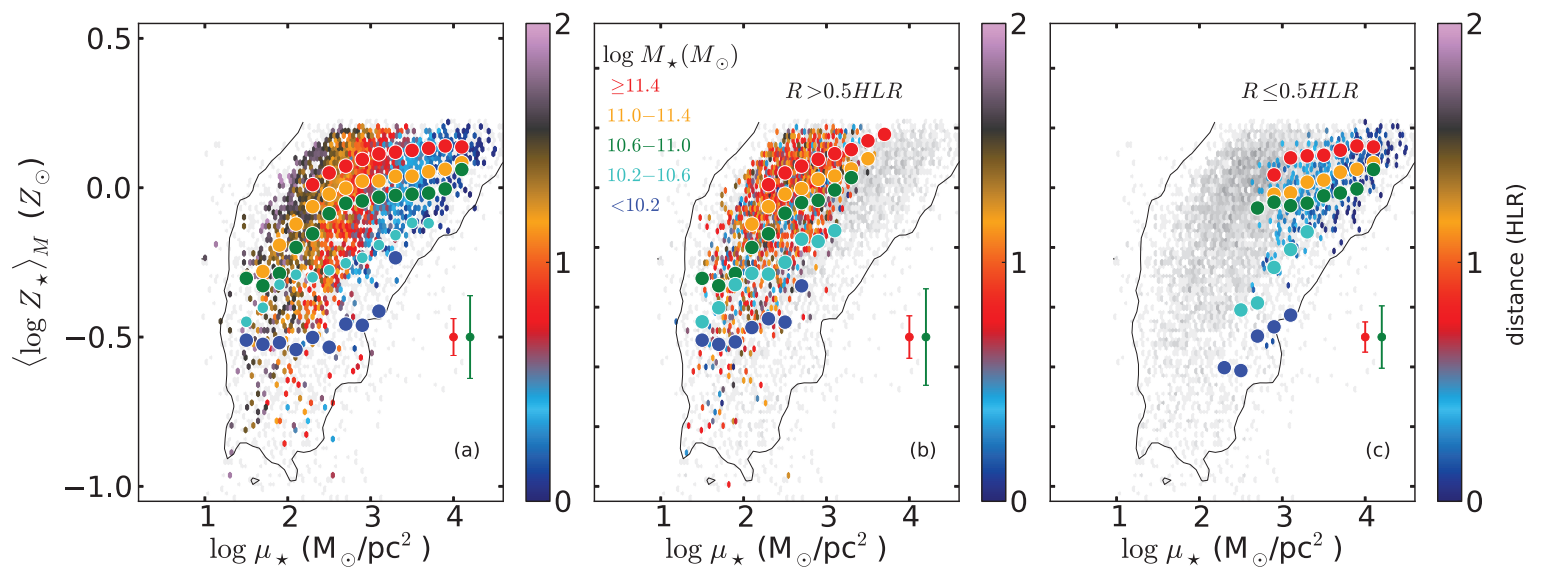

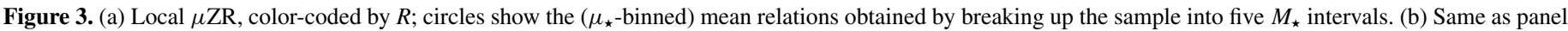

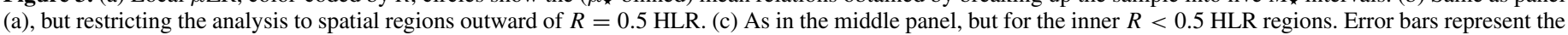
typical dispersion in metallicity in the local MZR in two of the five $M_{\star}$ intervals.

(A color version of this figure is available in the online journal.)

Clearly, despite the scatter, local stellar mass surface density and metallicity are strongly correlated, and in a radially dependent fashion. The general trend is depicted as gray circles, which averages over metallicities in 0.2 dex wide bins in $\mu_{\star}$.

The average $\mu \mathrm{ZR}$ traced by the gray circles in Figure 2(a) should not be confused with the global one, since $R$ bins do not contribute equally to the galaxy-wide averages of either $\mu_{\star}$ or $\left\langle\log Z_{\star}\right\rangle_{M}$. The $\left(\mu_{\star}\right.$-binned) global version of the $\mu \mathrm{ZR}$ is shown by the large green circles. Note that they follow closely the underlying orange/red points $(R \sim 1$ HLR in our color scheme), as expected from the fact that galaxy-wide averages essentially reflect properties at 1 HLR (GD14).

The global $\mu \mathrm{ZR}$ is visibly steeper than the local one, particularly in the high-density regime of the inner regions of galaxies (blueish points in Figure 2(a)), where the local $\mu \mathrm{ZR}$ (gray circles) flattens. This flattening is not picked up by the global relation, as there are no galaxies globally as dense as these central regions of spheroids.

Overall, we conclude that the $\mu \mathrm{ZR}$ shows the local stellar surface density to be an important driver of the stellar metallicity, except for their innermost densest regions, where some other property must take over the dominant role. Even in the outer regions, however, the vertical scatter in the $\mu \mathrm{ZR}$ is such that $\mu_{\star}$ cannot be the sole parameter regulating $Z_{\star}$.

\subsection{Stellar versus Nebular $\mu Z R s$ and Chemical Evolution}

Before furthering our investigation of global and local effects, we repeat in Figure 2(b) the exercise carried out in Figure 1(b) for the global MZR, but now for the $\mu \mathrm{ZR}$. The large gray circles are as in panel (a), but the blue ones track the relation obtained considering only young stars. Again, one obtains a flatter relation, indicative of chemical evolution. Also, as in Figure 1(b), the shape of the $\mu \mathrm{ZR}$ for young stellar populations approaches that obtained from $\mathrm{H}$ II regions (cyan-colored stars; Sánchez et al. 2013).

Figure 2(b) also summarizes how local SFHs vary across the $\mu \mathrm{Z}$ plane. This is revealed by the colors of the dots, which now indicate the radially averaged mean stellar age. As for the global MZR, one finds younger systems (i.e., those with more significant recent star formation) toward the low- $\mu_{\star}$, metal-poor corner of the $\mu \mathrm{ZR}$. Large ages, on the other hand, occur at the densest, more metal-rich regions typical of small $R$. These inner zones essentially completed their star formation and chemical evolution long ago. Note that this is also the regime where the local $\mu \mathrm{ZR}$ (gray circles) flattens.

\section{LOCAL + GLOBAL EFFECTS AND THE DISTINCT ROLES OF $\mu_{\star}$ AND $M_{\star}$ IN DISKS AND SPHEROIDS}

The results reported above send mixed messages as to what the main driver of stellar metallicities might be: while Figure 1 indicates that $M_{\star}$ is involved in establishing global $Z_{\star}$ values, Figure 2 points to $\mu_{\star}$ playing a major role in defining the local $Z_{\star}$. It thus seems that both local and global effects play relevant roles in determining the metallicity.

Figure 3 shows that this is indeed the case. The dots in all panels repeat the local $\mu \mathrm{ZR}$, but now breaking the mean (i.e., $\mu_{\star^{-}}$ binned) relation (large circles) into five approximately equally populated $M_{\star}$ intervals. Panel (a) shows results considering all 6000 radial bins, while panels (b) and (c) take into account only points outside and inside $R=0.5 \mathrm{HLR}$, respectively. The figure thus explores the whole $M_{\star}-\mu_{\star}(R)-Z_{\star}(R)-R$ landscape.

Comparing Figure 3(a) with the mean $\mu \mathrm{ZR}$ traced by gray circles in Figure 2, one immediately realizes that a pure $\mu \mathrm{ZR}$ analysis overlooks the important role of $M_{\star}$ in defining the metallicity scale. Mass is a major source of scatter in the $\mu \mathrm{ZR}$, with massive galaxies being locally (and globally) more chemically enriched than low-mass ones for the same $\mu_{\star}(R)$. Still, the rise of $Z_{\star}(R)$ with $\mu_{\star}(R)$ for constant $M_{\star}$ shows the relevance of local effects, particularly for low- and intermediate$M_{\star}$ systems.

Though revealing, the $M_{\star}$-dependent mean $\mu$ ZRs in Figure 3(a) do not distinguish the whereabouts within a galaxy, as disk and bulge/spheroid locations are treated on equal footing. A coarse way to discriminate between these morphological components is to define them by a $R>$ or $<0.5$ HLR criterion. Panel (b) in Figure 3 shows the $\mu \mathrm{ZR}\left(M_{\star}\right)$ for $R>0.5$ HLR. Except for the 41 ellipticals, this plot should be read as a $\mu \mathrm{ZR}\left(M_{\star}\right)$ for galactic disks. In stark contrast to Figure 3(a), no downward flattening is seen. Stellar metallicities increase steadily with $\mu_{\star}$, modulated by a $M_{\star}$-related amplitude.

The panorama changes in Figure 3(c), where only the inner zones are considered. For the three largest $M_{\star}$ ranges $\left(>10^{10.6} M_{\odot}\right)$, one sees a very weak dependence of $Z_{\star}$ on $\mu_{\star}$. Local effects thus seem of secondary importance in the 
dense regime of the inner regions of massive galaxies. The $M_{\star}<10^{10.6} M_{\odot}$ bins, however, still follow a strong $\left(M_{\star}-\right.$ modulated) $\mu \mathrm{ZR}$. Galaxies in these low- $M_{\star}$ bins are predominantly $(87 \%)$ late-type spirals $(\mathrm{Sc}-\mathrm{Sd})$, with small or nonexistent bulges (possibly resulting from secular disk evolution; Fisher \& Drory 2011), which explains their disk-like $\mu \mathrm{ZR}$.

\section{DISCUSSION}

The general picture one draws from these results is that both global $\left(M_{\star}\right.$-driven) and local $\left(\mu_{\star}\right.$-driven) effects are important in determining the stellar (and, by extension, also the nebular) metallicities in galaxies, and that the overall balance between these two varies with the location within a galaxy. Two clearly distinct regimes are identified: (1) one related to disks, where $\mu_{\star}$ (hence local physics) regulates metallicity, modulated by an $M_{\star}$-related amplitude, and (2) the other pertaining to the bulge/ spheroid component, where $M_{\star}$ dominates the physics of star formation and chemical enrichment.

These results tie in nicely with the recent analysis of GD14. We have shown there that mean stellar ages (a first moment descriptor of the SFH) relate strongly to $\mu_{\star}$ in galactic disks, indicating that local properties dictate the pace of star formation. The slower growth (hence younger ages) found at low $\mu_{\star}$ should lead to less metal enrichment, in agreement with our $\mu \mathrm{ZR}$. Within bulges/spheroids, $M_{\star}$ is a much more relevant driver of the SFH. Most of the star formation in these regions was finished long ago, leading to fast metal enrichment and little or no chemical evolution since those early days, as found in this Letter.

Despite the overall consistency and physical appeal of our results, there are some caveats. Aside from limitations of the method (e.g., Cid Fernandes et al. 2014), and the choice of $R=0.5$ to segregate bulges from disks, a more philosophical kind of caveat holds the promise of interesting future work. The goal of this Letter was to evaluate the role of global and local effects in determining stellar metallicities in and within galaxies. To this end, we have chosen $M_{\star}$ and $\mu_{\star}$ as the representatives of global and local effects, respectively, but was this a wise choice of variables?

Our answer at this stage is a "yes" for $M_{\star}$, but only a "perhaps" for $\mu_{\star}$. Both the SFH of a galaxy and its chemical evolution are thought to be affected by its mass, including its dark + gaseous + stellar components. In practice, $M_{\star}$ is the most readily available proxy for total mass, and thus a relevant variable.

The status of $\mu_{\star}$ as a tracer of local effects is less clear. The choice of $\mu_{\star}$ as an independent variable is inspired by Schmidt (1959), who postulated a density-controlled star formation rate law, and by the fact that our analysis provides robust estimates of $\mu_{\star}$. Clearly, $\mu_{\star}$ is but a proxy for complex star formation physics. The fact that we find $Z_{\star}$ to correlate strongly with $\mu_{\star}$ in disks suggests it to be a reasonable empirical proxy for local effects. Yet, the fact that the disk $\mu$ ZR has its amplitude modulated by $M_{\star}$ raises doubts as to how fundamental $\mu_{\star}$ really is.

While one can think of ways to relate local $Z_{\star}$ values to the global $M_{\star}$, there are other ways of formulating the problem. The $Z_{\star}$-scaling role of $M_{\star}$ in the disk $\mu \mathrm{ZR}$ may be reflecting the works of a different $M_{\star}$-related property, like morphological type, gas fraction, in/outflow rates, or others. Future work should explore these possibilities.

This Letter is based on data obtained by the CALIFA survey (http://califa.caha.es), funded by the Spanish MINECO grants ICTS-2009-10, AYA2010-15081, and the CAHA operated jointly by the Max-Planck IfA and the IAA (CSIC). The CALIFA Collaboration thanks the CAHA staff for the dedication to this project. Support from CNPq (Brazil) through Programa Ciência sem Fronteiras (401452/2012-3) is duly acknowledged.

\section{REFERENCES}

Bell, E. F., \& de Jong, R. S. 2000, MNRAS, 312, 497

Cid Fernandes, R., Asari, N. V., Sodré, L., et al. 2007, MNRAS, 375, L16

Cid Fernandes, R., González Delgado, R. M., Pérez, E., et al. 2014, A\&A, 561,130

Cid Fernandes, R., Mateus, A., Sodré, L., Stasińska, G., \& Gomes, J. M. 2005, MNRAS, 358, 363

Cid Fernandes, R., Pérez, E., García Benito, R., et al. 2013, A\&A, 557, 86

Faber, S. M., \& Jackson, R. E. 1976, ApJ, 204, 668

Fisher, D. B., \& Drory, N. 2011, ApJL, 733, L47

Gallazzi, A., Charlot, S., Brinchmann, J., White, S. D. M., \& Tremonti, C. A. 2005, MNRAS, 362, 41

González Delgado, R. M., Cerviño, M., Martins, L. P., Leitherer, C., \& Hauschildt, P. H. 2005, MNRAS, 357, 945

González Delgado, R. M., Pérez, E., Cid Fernandes, R., et al. 2014, A\&A, 562,47

Husemann, B., Jahnke, K., Sánchez, S. F., et al. 2013, A\&A, 549, A87

Iglesias-Páramo, J., Vílchez, J. M., Galbany, L., et al. 2013, A\&A, 553, L7

Mast, D., Rosales-Ortega, F. F., Sánchez, S. F., et al. 2014, A\&A, 561, 129

Panter, B., Jimenez, R., Heavens, A. F., \& Charlot, S. 2007, MNRAS, 378, 1550

Panter, B., Jimenez, R., Heavens, A. F., \& Charlot, S. 2008, MNRAS, 391, 1117

Pérez, E., Cid Fernandes, R., González Delgado, R. M., et al. 2013, ApJL, 764, L1

Rosales-Ortega, F. F., Sánchez, S. F., Iglesias-Páramo, J., et al. 2012, ApJL, 756, L31

Sánchez, S. F., Kennicutt, R. C., Gil de Paz, A., et al. 2012, A\&A, 538, A8

Sánchez, S. F., Rosales-Ortega, F. F., Jungwiert, B., et al. 2013, A\&A, 554, 58

Schmidt, M. 1959, ApJ, 129, 243

Tremonti, C. A., Heckman, T. M., Kauffmann, G., et al. 2004, ApJ, 613, 898

Vazdekis, A., Sánchez-Blázquez, P., Falcón-Barroso, J., et al. 2010, MNRAS, 404, 1639

Walcher, C. J., Wisotzki, L., Bekeraité, S., et al. 2014, A\&A, in press (arXiv:1407.2939) 